\title{
Cytomegalovirus Encephalitis with Decreased Vision in Immunocompromised Patients Treated with Valganciclovir: Case Report
}

\author{
Erwin Hardiansyah ${ }^{1}$, Paulus Sugianto ${ }^{2}$ \\ ${ }^{1}$ Neurology Resident of Faculty of Medicine, Airlangga University/Dr. Soetomo General Hospital Surabaya \\ ${ }^{2}$ Staff of Department Neurology, Faculty of Medicine, Airlangga University/Dr. Soetomo General Hospital \\ Surabaya \\ Corresponding Author: Erwin Hardiansyah
}

\begin{abstract}
Introduction: Cytomegalovirus (CMV) is a significant cause of morbidity and mortality in patients with Acquired Immune Deficiency Syndrome (AIDS). Almost all Human Immunodeficiency Virus (HIV) infected patients develop CMV rapidly with manifestation in the central nervous system. Most CMV encephalitis is preceded by the presence of CMV in other places. Therefore, immediate therapy will be more effective and the delay in therapy can be detrimental.

Case: A 40-year-old man with the major complaint of vision decrease in both eyes for 3 months claimed that he felt worse after 7 days, accompanied by headache and fever. This patient was diagnosed with HIV 2 years ago. The examination obtained GCS 456, negative meningeal sign, headache with NRS 7. The visual acuity of the right eye was negative light perception and in the left eye of vision 1/300. The patient experienced clinical improvement after oral Valganciclovir therapy with a dose of 2x450 mg.
\end{abstract}

Conclusion: CMV encephalitis must be considered to diagnose earlier in HIV patients with complaints of decreased vision. With valganciclovir $2 \times 450 \mathrm{mg}$ tablets improve reduction of headache scale NRS from 7 to 2 , increasing left eye vision from $1 / 300$ to $6 / 60$.

Keywords: Encephalitis CMV, HIV, Valganciclovir

\section{INTRODUCTION}

Cytomegalovirus (CMV) infection most commonly infects humans worldwide usually without obvious symptoms. CMV infection first attracted medical attention with the identification of owl's eye inclusions on microscopic examination of newborns in 1910 and in 1964 among patients receiving organ transplants. More modern diagnostic methods show that CMV is an opportunistic infection commonly found in fetuses, allograft recipients, patients receiving bone marrow transplantations, and AIDS patients. CMV infection is associated with transplant outcomes, including graft rejection response and cardiovascular disease. ${ }^{1}$

Cytomegalovirus (CMV) is a major cause of morbidity and mortality in patients with Acquired Immune Deficiency Syndrome (AIDS). ${ }^{2}$ Almost all HIVinfected patients develop CMV rapidly with manifestations in the central nervous system. The introduction of Highly Active Antiretroviral Therapy (HAART) improved the prognosis of HIV-infected patients and had a profound impact on the incidence of CMV disease. ${ }^{2}$

Immunocompetence in AIDS patients is primarily based on the level of the HIV virus and the level of their T-helper cells, particularly CD4 + T-lymphocytes that are attacked by the HIV virus. CMV is most opportunistic when the CD4 cell is less 
than 50 and is rarely seen in those with more than 200. Normal T-lymphocyte levels range from 400 to 1700 cells/ul in healthy patients. $^{3}$

The most common clinical manifestations of $\mathrm{CMV}$ reactivation are retinitis (about $80 \%$ of cases), ${ }^{6,4,5}$ and gastrointestinal diseases (colitis, esophagitis, and gastritis) in about $15 \%$ of cases. Less common manifestations are encephalitis, pneumonia, polyradiculopathy, adrenalitis, sclerosing cholangitis, and hepatitis. ${ }^{4}$

Cytomegalovirus retinitis is the most common ocular opportunistic infection, representing $90 \%$ of infectious retinitis, 5,6 $20-30 \%$ of patients with AIDS develop CMV retinitis. This usually occurs in the late stages of disease (approximately 18 months after declaration of clinical onset) in patients with a lower CD4 count of $50 /$ mmc. $^{5}$ Single or total blindness due to HIV/AIDS is generally a deep immune suppression disease (CD4 T-lymphocyte count $<50$ cells $/ \mathrm{mm} 3),{ }^{7}$ most commonly caused by CMV retinitis. Without treatment, life time after a diagnosis of CMV retinitis can be measured in weeks or months. ${ }^{8}$

The Human Immunodeficiency Virus (HIV) has the ability to affect any organ in the body. In $70 \%$ of HIV-infected patients, ocular manifestations are observed, most of which reflect systemic disease and maybe the first sign of disseminated infection. $^{7}$ Patients infected with HIV during the active phase of viral replication have a 5.2-51\% risk of CMV infection, with a median value of $20 \%$. The risk factor is a progressive loss of immune function with a CD4 lymphocyte count less than 100/1L. ${ }^{9}$

\section{CASE REPORT}

A 40-year-old man with the major complaint of gradually decreased vision in both eyes for 3 months claimed that he felt worse after 7 days, accompanied by headache and fever. This patient was diagnosed with HIV 2 years ago, received Highly Active Antiretroviral Therapy (HAART) therapy with Lamuvudine 150 mg, Zidovudin 300 mg, Nevirapine 200 mg twice a day, but the patient did not take his medications regularly. Risk factors were found on the patient in the form of drug abuse and multi-partner sex activities. The physical examination obtained Glasgow Coma Scale (GCS) of 456, negative meningeal sign, headache with the Numeric Rating Scale (NRS) 7, visual acuity of the right eye was negative light perception and visual acuity of the left eye was $1 / 300$, CD 4 10 cells/uL, IgM CMV:1,15 Ua/ml, IgG CMV: $54 \mathrm{Ua} / \mathrm{ml}$. Lumbar Puncture: clear color, total cell of $0.015103 / \mathrm{ul}$, protein of $33.7 \mathrm{mg} / \mathrm{dl}$, MN 100\%, Nonne (-), Pandy (), Glucose of $33 \mathrm{mg} / \mathrm{dl}$. PCR CMV Liquor Cerebrospinalis (LCS) was Positif. The patient underwent a fundus photo examination before therapy (figure 1). After being treated for 21 days, the patient had a fundus photo taken to evaluate the progress of the therapy that had been given (figure 2).

The patient had received treatment and was evaluated for 21 days. The patient experienced clinical improvement after consuming Valganciclovir $2 \times 450 \mathrm{mg}$ therapy. The improvements were in the form of a decrease in the headache scale from NRS 7 to NRS 0-2 and an increase in left eye vision from $1 / 300$ to $6 / 60$

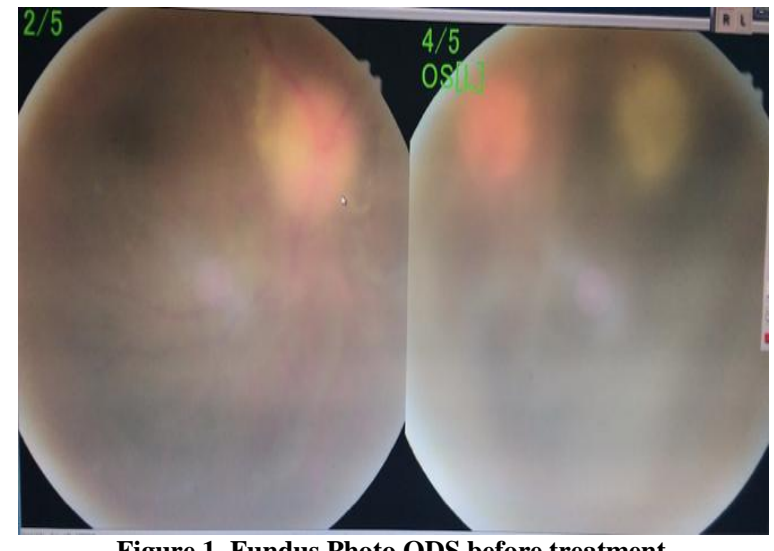

Figure 1. Fundus Photo ODS before treatment OD: Fundus reflex (+), papilla $N$ II is difficult to evaluate because of the opacity of the vitreous body, which gives a clear impression. Color, retina are difficult to evaluate, reflex macula is difficult to evaluate.

OS: Fundus reflex (+), papilla N II is difficult to evaluate because of the opacity of the vitreous body, giving the impression of a firm boundary. Color is difficult to evaluate. The retina is difficult to evaluate, the impression obtained of exudate on the macula can be a sign of active infection of the retina. 


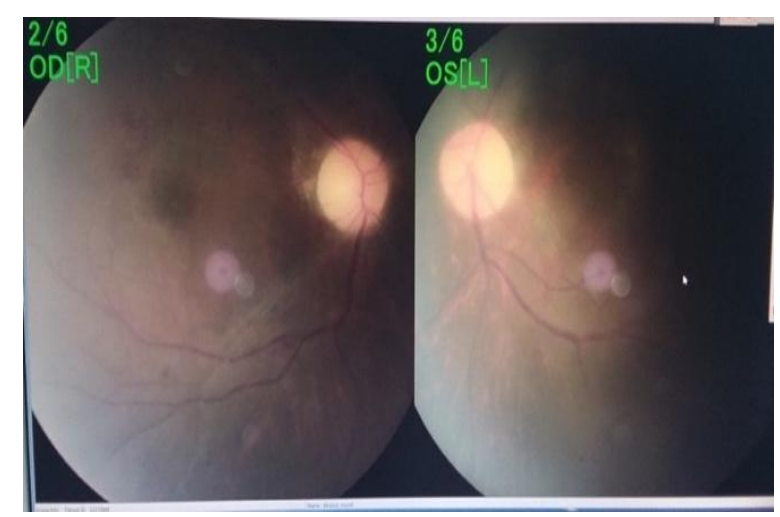

Figure 2. Fundus Photo After Treatment ODS papillae atrophy, OS does not show macular exudate (which was found on previous examinations) indicating an improvement/ response to therapy. ODS Decreased vitreous body opacification.

\section{DISCUSSION}

From the patient's anamnesis, there was the occurrence of headache, fever, and vomiting. Furthermore, the patient also complained of vision decrease in the right eye for the past 7 days, after previously suffering from gradual vision decrease in the left eye for the last 3 months. The patient diagnosed with HIV 2 years ago had received ART therapy but did not take his medication regularly.

The symptom characteristics in this patient were consistent with the clinical picture of CMV encephalitis with CMV retinitis, whereas CMV encephalitis often affects immunocompromised patients and rarely immunocompetent patients.

Most people who are infected by the virus do not show significant symptoms. The symptoms are similar to common flu and last for 2-3 weeks. However, the manifestations of viral infections vary from mild to life-threatening, even causing death. Most people with mild cases make a full recovery. However, those with severe cases, even though they recover, will sustain permanent damage to their nervous system. ${ }^{10}$

On the examination, the visual acuity in the right eye was negative and the visual acuity in the left eye was $1 / 300$, and the visual field was normal. In this case, we consulted the patient to an ophthalmologist for an ophthalmological examination, and then he was diagnosed with CMV rhinitis. In a review conducted by Arribas et al. on
676 patients with CMV encephalitis, it was said that as many as $85 \%$ of patients were infected with HIV; $12 \%$ had immunosuppression due to other causes (organ transplant). Then, only 3\% were immunocompetent. Most patients with CMV encephalitis have pre-existing CMV disease elsewhere, such as the retina. CMV retinitis is a common problem for people with AIDS. People with HIV or AIDS still have a higher risk for CMV retinitis. Recent autopsy studies have shown that as many as $42 \%$ of patients with CMV rhinitis have CMV encephalitis; therefore, ophthalmological examinations are required in AIDS patients with CMV encephalitis. ${ }^{11}$

A study from the United States found that CMV retinitis was bilateral in $28 \%$ of newly diagnosed cases before taking ART, and only $26 \%$ of new cases diagnosed after the introduction of ART. Furthermore, among the latter group, involvement was only 9\% among patients who had never used ART, suggesting that second eye involvement was a late occurrence in the course of CMV retinitis. ${ }^{12}$

The supporting investigation, it was found that CD4 10 cells/uL and $\operatorname{IgM} \operatorname{IgG}$ CMV was positive. Furthermore, on analysis of CSF with viral encephalitis results, CMV PCR was positive. Infection of the Central Nervous System is often a complication in patients with advanced HIV and usually occurs when the CD4 is less than 100 cells/cmm. Multivariate analysis showed that the PCR method was superior to other tests, namely antigenemia and CMV culture (odds ratio: CMV PCR 10.0; antigenemia test and CMV culture 4.3). According to Tunkel AR et al., there were studies on 99 patients in which stated that the CMV culture results from plasma and urine were compared with plasma PCR, where plasma PCR was superior to CMV culture to identify AIDS patients at risk for CMV disease. Plasma PCR examination can measure CMV very accurately, however, the sensitivity is low at low numbers, cerebrospinal PCR for CMV (for immunocompromised patients is $82 \%$ - 
$100 \%$; specificity $86 \%-100 \%) .{ }^{3}$ Positive PCR test in cerebrospinal fluid indicates CMV neuroinfection and correlated with poor prognosis. $^{13}$

Studies in AIDS patients with CD4 counts $<50-100$ cells/cmm illustrate a correlation between various symptoms of Central Nervous System (CNS) disorders consistent with myelitis or encephalitis, CSF lymphocytic lymphocytosis, elevated CSF protein, and virus in the CNS that can be detected by antigens pp65 or nucleic amplification examination. In most reports, evidence of viral replication in the CSF compartment is essential for the diagnosis of CMV encephalitis. ${ }^{2}$

The patient experienced clinical improvement after consuming Valganciclovir $2 \times 450 \mathrm{mg}$ orally. The improvements were in the form of a decrease in the headache scale from NRS 7 to NRS 2 and an increase in left eye vision from $1 / 300$ to $6 / 60$.

The first-line options for the treatment of CMV disease are almost always intravenous Ganciclovir or oral Valganciclovir. The earliest major study demonstrated the non-inferiority of oral Valganciclovir. Intravenous Valganciclovir was administered to AIDS patients with CMV retinitis, and oral Valganciclovir was found to be as effective as intravenous Ganciclovir as induction therapy. Large RCTs have compared oral Valganciclovir and intravenous Ganciclovir for the treatment of CMV disease in Solid Organ Transplantation (SOT) recipients. The patients were randomised treated with oral Valganciclovir $900 \mathrm{mg}$ or intravenous Ganciclovir $5 \mathrm{mg} / \mathrm{kg}$ twice daily for 21 days, followed by Valganciclovir $900 \mathrm{mg}$ daily for 28 days. Viremia clearance on the 21 st day was $45 \%$ in the oral Valganciclovir group and $48 \%$ in the arm intravenous Ganciclovir group. The treatment success on the 49th day was $85 \%$ in the oral Valganciclovir group and $84 \%$ in the arm intravenous Ganciclovir group. ${ }^{4}$

The optimal treatment of CMV disease of the CNS has not been well established. Guidelines for the treatment of CMV disease in AIDS patients recommend the use of Ganciclovir or Foscarnet, as well as consideration of therapy combination for patients who have been receiving treatement. $^{2}$

\section{CONCLUSION}

Cytomegalovirus (CMV) infection is very high in prevalence worldwide. CMV encephalitis mainly affects immunocompromised patients and rarely the immunocompetent. Most patients with CMV encephalitis have pre-existing CMV disease elsewhere, such as the retina.

Patients with HIV who undergo HAART therapy should be monitored and evaluated during the therapy, including adherence to medication, complaints and clinical conditions of patients, as well as evaluation of $\mathrm{CD} 4$ to prevent other complications of the disease. If there is a decrease in clinical condition accompanied by complaints of new neurological deficits, the doctor must quickly conduct a physical examination and support for immediate diagnosis of complications of the patient's disease. Therapy will be effective if it is started immediately, otherwise, delays in therapy can be detrimental.

The patient had received oral Valganciclovir with a dose of $2 \times 450 \mathrm{mg}$ per taking, monitored, and clinically evaluated for 21 days, in which clinical improvement was found in the form of a decrease in pain scale from NRS 7 to NRS 2, and there was also an increase in visual acuity in the left eye from visual acuity of $1 / 300$ to $6 / 60$. Further, in the eyes evaluation, we conducted a photo ophthalmological examination, the comparison of results before and after 21 days of therapy showed improvements.

\section{Acknowledgement: None}

Conflict of Interest: The authors affirm no conflict of interest in the study.

\section{Source of Funding: None}




\section{REFERENCES}

1. Hartanto,Q S. Infeksi Pada Sistim Saraf Edisi 2 Kelompok Studi Neuro Infeksi Perdossi. Paulus Sug. (Sugianto P, Mawuruntu, H P, eds.). Surabaya: Airlangga University Press; 2020.

2. Baghban A, Malinis M. Ganciclovir and foscarnet dual-therapy for cytomegalovirus encephalitis: A case report and review of the literature. J Neurol Sci. 2018;388 (January): 28-36. doi:10.1016/j.jns.2018.02.029

3. Conlin KA. Management of Cytomegalovirus Retinitis in a Patient with AIDS and Poor Compliance with HAART Therapy. 2001.

4. Tan BH. Cytomegalovirus Treatment. Curr Treat Options Infect Dis. 2014;6(3):256270. doi:10.1007/s40506-014-0021-5

5. Chiotan C, Radu L, Serban R, Cornăcel C, Cioboata M, Anghel A. Cytomegalovirus retinitis in HIV/AIDS patients. J Med Life. 2014;7(2):237-240.

http://www.ncbi.nlm.nih.gov/pubmed/25408 732\%0Ahttp://www.pubmedcentral.nih.gov/ articlerender.fcgi?artid=PMC4197487.

6. Gupta R. Cytomegalovirus retinitis: A case report. Med J Dr DY Patil Univ. 2014;7(6): 822. doi:10.4103/0975-2870.144901

7. Lee $\mathrm{CH}$, Bright DC, Ferrucci S. Treatment of cytomegalovirus retinitis with oral valganciclovir in an acquired immunodeficiency syndrome patient unresponsive to combination antiretroviral therapy. Optometry. 2006;77(4):167-176. doi:10.1016/j.optm.2006.01.019

8. Guex-Crosier Y, Telenti A. An epidemic of blindness: A consequence of improved HIV care? Bull World Health Organ. 2001;79(3): 181.
9. Styczynski J. Who Is the Patient at Risk of CMV Recurrence: A Review of the Current Scientific Evidence with a Focus on Hematopoietic Cell Transplantation. Infect Dis Ther. 2018;7(1):1-16. doi:10.1007/s40121-017-0180-z

10. Rianawati S. KELOMPOK STUDI NEURO INFEKSI. (Sugianto P, Sudewi R, Ritawang $\mathrm{K}$, eds.). Surabaya: Airlangga University Press; 2011.

11. Puspitarini D, Hartono H. Ensefalitis Cytomegalovirus. 2015.

12. Ford N, Shubber Z, Saranchuk P, et al. Burden of HIV-Related cytomegalovirus retinitis in resource-limited settings: A systematic review. Clin Infect Dis. 2013; 57(9):1351-1361. doi:10.1093/cid/cit494

13. M. P-Ś, A. N, M. G, et al. Recommendations for the diagnosis and treatment of CMV infections. Polish Society of Epidemiology and Infectious Diseases. Przegl Epidemiol. 2016;70(2):297-310. http://www.embase.com/search/results?suba ction $=$ viewrecord \&from $=$ export\&id $=\mathrm{L} 6167$ 25791\%0Ahttp://vu.on.worldcat.org/atoztitl es/link?sid=EMBASE\&issn=00332100\&id $=$ doi: $\&$ atitle $=$ Recommendations + for + the + di agnosis+and+treatment+of+CMV+infection s. + Polish + Society+of+Ep.

How to cite this article: Hardiansyah E, Sugianto P. Cytomegalovirus encephalitis with decreased vision in immunocompromised patients treated with valganciclovir: case report. International Journal of Research and Review. 2021; 8(9): 149-153. DOI: https://doi.org/10. 52403/ijrr.20210921 